\title{
Modelling charge transport in organic semiconductors: from quantum dynamics to soft matter
}

\author{
David L. Cheung and Alessandro Troisi
}

Received 7th May 2008, Accepted 26th June 2008

First published as an Advance Article on the web 26th August 2008

DOI: $10.1039 / \mathbf{b 8 0 7 7 5 0 a}$

The charge carrier dynamics in organic semiconductors has been traditionally discussed with the models used in inorganic crystalline and amorphous solids but this analogy has severe limitations because of the more complicated role of nuclear motions in organic materials. In this perspective, we discuss how a new approach to the modelling of charge transport is emerging from the alliance between the conventional quantum chemical methods and the methods more traditionally used in soft-matter modelling. After describing the conventional limit cases of charge transport we discuss the problems arising from the comparison of the theory with the experimental and computational results. Several recent applications of numerical methods based on the propagation of the wavefunction or kinetic Monte Carlo methods on soft semiconducting materials are reviewed.

\section{Introduction}

The idea of using organic conjugated materials as the active elements for electronic devices started circulating only few years after the development of the first electronic devices based on inorganic semiconductors ${ }^{1}$ but, for the majority of the past 60 years, it did not translate into a useful technology, overshadowed by the success and the technological advances of inorganic semiconductor industry. Interest in organic semiconductors research was revived during the 1990s thanks to the combination of two factors. First, a set of crucial experiments demonstrated the feasibility of cost-effective organic light emitting diodes (LED) $)^{2-4}$ and thin film transistors $(\mathrm{TFT})^{5,6}$ followed few years later by the development of the first prototypes of organic photovoltaic (PV) devices. ${ }^{7-9}$ Secondly, the demand for low-cost and large-area semiconducting devices increased approximately in the same period, stimulated by the greater distribution of electronic devices and

Department of Chemistry and Centre of Scientific Computing, University of Warwick, Coventry, UK CV4 7AL

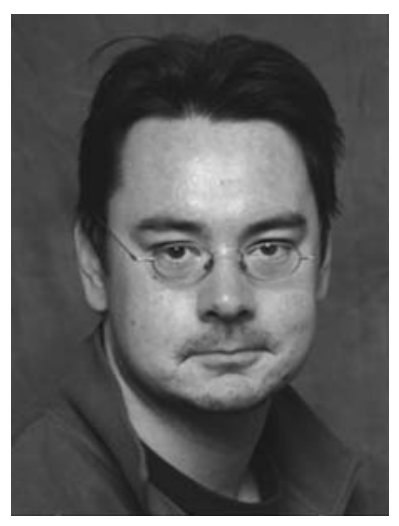

David Cheung
David Cheung has been involved in computer simulation of liquid crystals for several years. Following his PhD at the University of Durham, working in the group of Dr Mark Wilson, he moved to the University of Bielefeld to work with Prof. Fredierike Schmid. He then moved to the University of Warwick, first to work with Prof. Mike Allen, then to the group of Dr Troisi.

display and the need to develop new efficient photovoltaic cells and energy efficient lighting. While organic LEDs are already found on the market, ${ }^{10,11}$ the development of suitable TFT and PV devices is still subject of fundamental investigation involving the development of new materials, processing ${ }^{12}$ and device architectures. ${ }^{13}$

There are two major classes of materials which have been considered somewhat separately in the field of organic electronics. Crystalline molecular semiconductors (some of which are illustrated in Fig. 1), which have been studied in bulk and thin film with a variety of techniques, offer the advantages of a well defined structure and, in principle, a simple identification of the structure-property relation. ${ }^{14-20}$ Polymeric semiconductors, on the other hand, are potentially simpler to process and appear to be more suitable for 'printed' electronics. ${ }^{21}$ Isotropic polymers (e.g. polytriaryamine) are more frequently used in LED, while highly ordered (anisotropic) compounds, for example poly(3-hexylthiophene) or (P3HT), are considered more suitable for thin film transistors. ${ }^{22}$ Liquid crystalline ${ }^{23}$ and amorphous ${ }^{4}$ molecular semiconductors are also actively investigated and tend to have similar characteristics of charge

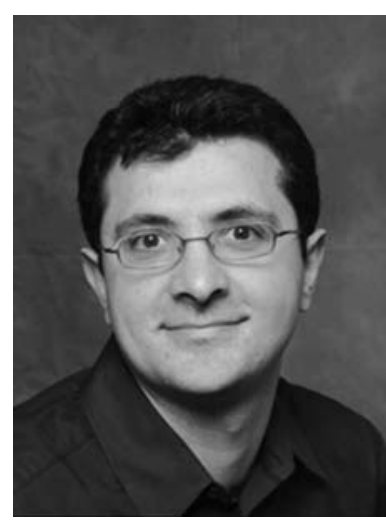

Alessandro Troisi
Alessandro Troisi received his $\mathrm{PhD}$ from University of Bologna in 2002. He was a postdoctoral researcher at the Northwestern University and Bologna and he joined the University of Warwick in 2005. His research interests include electron transport in organic materials, singlemolecule electric junctions, and molecular self-assembly. In 2007 he was awarded the Marlow Medal from the Royal Society of Chemistry. 

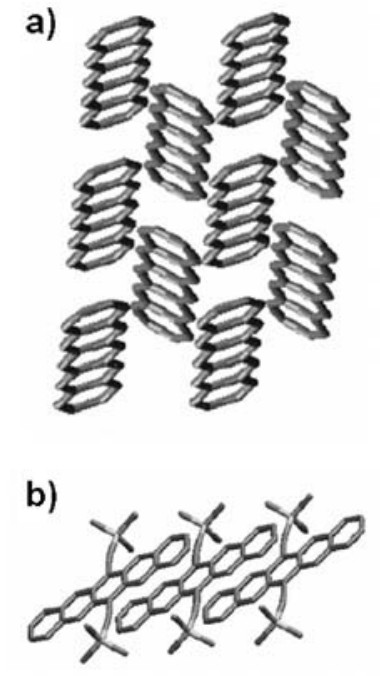
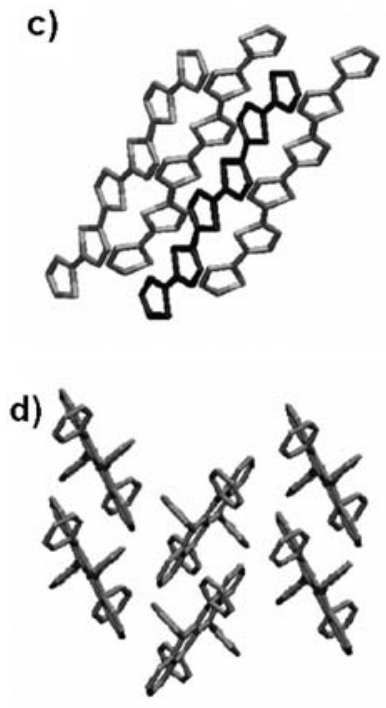

Fig. 1 Illustration of the crystal packing of four among the most studied crystalline organic semiconductors: (a) pentacene, (b) TMS pentacene derivative, ${ }^{20}$ (c) hexthiophene and (d) rubrene (hydrogen atoms are not represented).

transport and processability of polymeric materials. The most important characteristics for all organic semiconductors is the charge mobility, which is generally much lower than conventional semiconductor like silicon. A minimum acceptable value of mobility for a TFT is $1 \mathrm{~cm}^{2} \mathrm{~s}^{-1} \mathrm{~V}^{-1}$, while the best results obtained with polymeric materials is one order of magnitude lower. ${ }^{24}$ Record mobilities exceeding $10 \mathrm{~cm}^{2} \mathrm{~s}^{-1} \mathrm{~V}^{-1}$ have been reported for highly purified single crystals of pentacene ${ }^{17}$ and rubrene. $^{25}$

Theory is expected to play a major role in the search of new organic materials with improved mobility, by rationalizing the available results and guiding the synthesis of new materials. However, notwithstanding the great accuracy in the computation of the electronic and vibrational structure of the relevant materials, it is still not possible to predict the relative mobility of a set of related compounds. The difficulty in deriving a clear structure-property relation is due to the lack of a sound understanding of the charge transport mechanism in organic semiconductors. For a long time, attempts were made to understand organic semiconductors using theories and methods analogous to those used for inorganic semiconductors and it became clear only in the past few years that this analogy can be rather misleading. The transport in crystalline pentacene cannot be described as simple hopping between localized sites or by a band transport model without contradicting part of the experimental evidence.

If one is not interested in the relation between the chemical structure and the electric properties, it is possible to describe the device using phenomenological theories, which assume a simplified model of transport and derive a relation between observable quantities (current or mobility, voltage or electric field, temperature) and a few parameters that can be used to fit the experimental results. The Gaussian disorder model $(\mathrm{GDM}),{ }^{26}$ its recent improvements, ${ }^{27-29}$ and the multiple trapping model, ${ }^{30}$ are prototypes of this modelling paradigm

(based on the early work of Mott $^{31}$ on disordered inorganic materials). These methods have found many applications for the modelling of highly disordered organic solids ${ }^{32}$ and polymeric material ${ }^{33,34}$ and they are particularly useful for (mesoscale) electric device modelling. ${ }^{35}$

It might seem that the modelling of transport in crystalline materials (mostly based on microscopic theories and realistic Hamiltonians) and in polymeric materials (mostly based on phenomenological theories) are bound not to meet at any point. On the contrary, recent experimental and theoretical studies indicate that the difference between crystalline and polymeric materials is more subtle than previously thought. Many studies have shown that crystalline organic solids, held together by weak Van der Waals forces, are relatively soft and this softness is likely to affect dramatically the charge transport. The majority of high mobility polymeric materials display a high level of ordering and the correlation between high order and high mobility is very well established. ${ }^{36-38}$ In the past few years, several research groups started combining the investigation of the electronic structure of organic materials with the study of their nuclear dynamics, traditionally of interest only for the mechanical and rheological properties. Quantum chemical methods have been combined with classical molecular dynamics methods to attempt a multiscale description of the dynamic and static disorder in the organic material and the electron dynamics.

In this perspective we describe how this new approach to the modelling of charge transport in organic materials is emerging from the alliance between conventional methods of solid state physics and quantum chemistry with those traditionally used in soft-matter modelling. As we will see, the interaction between nuclear motions and electronic motions at different timescales is crucial for the description of the charge dynamics of all types of organic semiconductors. For convenience of exposition we will separate the discussion on crystalline solids (presented in section 2, including an outline of the conventional transport models) and the study of polymer and liquid crystalline materials (presented in section 3). A discussion of the challenges for the forthcoming years is presented in the last section.

\section{Modelling charge transport in molecular crystals}

\subsection{Two idealized cases of transport}

Any transport mechanism can be seen as an idealization, i.e. a minimalist representation of the system Hamiltonian that allows the description of the charge carrier dynamics. The most common idealization of a molecular crystal is that of an array of molecules, with one state per molecule, each one coupled with its neighbours, whose purely electronic Hamiltonian is usually written as: $:^{39,40}$

$$
H_{\mathrm{el}}=\sum_{j} \varepsilon_{j} a_{j}^{\dagger} a_{j}-\tau \sum_{j} a_{j}^{\dagger} a_{j+1}
$$

Here $a_{j}^{\dagger}$ and $a_{j}$ are the creation an annihilation operators of a charge carrier on site $j, \varepsilon_{j}$ is the energy of the carrier isolated on site $j$, and $-\tau$ is the electronic coupling between states localized on adjacent molecules. The molecule is further idealized as 
having only one vibrational degree of freedom, not coupled to the vibrations of the other molecules so that the phonons of the one-dimensional systems are dispersionless with energy $\hbar \omega_{0}$. They can be described as localized vibrations and the phonon Hamiltonian reads:

$$
H_{\mathrm{phon}}=\sum_{j} \hbar \omega_{0}\left(b_{j}^{\dagger} b_{j}+\frac{1}{2}\right)
$$

where $b_{j}^{\dagger}$ and $b_{j}$ are the creation and annihilation operators for the phonon. When a charge carrier (electron or hole) is present on one isolated molecule, the latter is deformed along its vibrational mode and the energy reduced by $\frac{1}{2} \hbar \omega_{0} g^{2} . g$ is the local or Holstein electron phonon coupling constant and the Hamiltonian representing this coupling is written as:

$$
V_{\mathrm{el}-\mathrm{phon}}^{\text {local }}=\sum_{j} g \hbar \omega_{0}\left(b_{j}^{\dagger}+b_{j}\right) a_{j}^{\dagger} a_{j}
$$

The strength of the Holstein electron-phonon coupling is often discussed in terms of the reoganization energy $\lambda=$ $\hbar \omega_{0} g^{2}$. The total Hamiltonian of the system can be therefore written as:

$$
H=H_{\mathrm{el}}+H_{\text {phon }}+V_{\mathrm{el}-\mathrm{phon}}^{\text {local }}
$$

There are two extreme cases which form the basis for our understanding of charge transport mechanism in conventional inorganic solids.

(i) If $V_{\text {el-phon }}^{\text {local }}$ can be neglected, electrons and phonons are decoupled and the charge carrier wavefunction is described by band theory. The charge carrier dynamics inside a band is satisfactorily treated by semiclassical theories ${ }^{41,42}$ according to which a delocalized wave packet is scattered by impurities or phonons with some characteristic scattering probability function. The carrier mobility $\mu$ in a band decreases with increasing temperature $T$ (as the scattering probability by phonons increases) with a characteristic power law $\mu \sim T^{-\alpha}$. The mobility in this case will be proportional to the intermolecular electronic coupling $\tau$, or, equivalently, to the inverse of the effective mass. Qualitatively not dissimilar is the case of weak

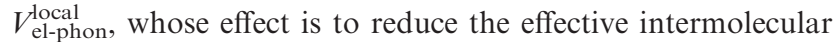
coupling to $\tau_{\text {eff }}=\tau \exp \left(-g^{2} \operatorname{coth}\left(\hbar \omega_{0} / 2 k_{\mathrm{B}} T\right)\right)$, ${ }^{39}$ i.e. to cause the charge carried dressed with phonons (called polaron) to be described by a renormalized (narrower) band. If $V_{\text {el-phon }}^{\text {local }}$ sufficiently small the carriers are still delocalized and the mobility decreases with increasing temperature following a power law.

(ii) If $\tau$ is very small (i.e. the intermolecular coupling can be treated as a perturbation) the localizing term $V_{\text {el-phon will }}^{\text {local }}$ dominate over the delocalizing term $-\tau \sum_{j} a_{j}^{\dagger} a_{j+1}$ and, at any given time, the charge will reside on one particular molecule. The mobility is due to thermally activated hopping from one molecule to another (incoherent transport). The rate of the hopping is often expressed using the simplified formula: ${ }^{39,43}$

$$
k_{\text {hop }}=\frac{\tau^{2}}{\hbar}\left(\frac{\pi}{k_{\mathrm{B}} T \lambda}\right)^{1 / 2} e^{-\lambda / 4 k_{\mathrm{B}} T}
$$

Which is valid in the rather restrictive conditions that $|\tau| \ll \lambda$ and $\hbar \omega_{0} \ll k_{\mathrm{B}} T$. We shall notice that both conditions are quite unrealistic for organic crystals, but more general expressions are available. ${ }^{44,45}$

\subsection{The computation of the Hamiltonian parameters and their rationalization}

It is clear from the discussion above that the key parameters for the computation of the mobility, according to the elementary models described above, are the intermolecular coupling $\tau$ and the reorganization energy $\lambda$. The computational evaluation of these two quantities, pioneered by the group of Bredas, ${ }^{46,47}$ allowed an important step forward in the understanding of these materials. It was in fact revealed that estimates of the parameters of the Hamiltonians based solely on the fitting of experimental mobility data led to unrealistic results. ${ }^{48}$ Moreover, even if the transport mechanism is not generally known, the mobility is expected to increase with the increase of the intermolecular coupling and is expected to decrease as the reorganization energy increases. In other words, there is the possibility to correlate, albeit not quantitatively, the Hamiltonian parameters and the measured mobility even in the absence of a well established transport mechanism.

The electronic coupling $\tau_{i j}$ between two molecular orbitals $\varphi_{i}$ and $\varphi_{j}$ residing on neighbouring molecules can be defined as:

$$
\tau_{i j}=\left\langle\varphi_{i}\left|H_{\mathrm{el}}\right| \varphi_{j}\right\rangle
$$

where $H_{\mathrm{el}}$ is the effective one electron Hamiltonian of the crystals, which can be identified with the Fock operator (in Hartree-Fock theory) or the Kohn-Sham-Fock operator (from DFT). The integral coupling in (6) can be computed explicitly, ${ }^{49}$ or from the 'energy splitting' method. ${ }^{50}$ According to the latter, if the isolated molecular orbital levels are sufficiently distant from one another, the levels of a dimer (with the molecules in the same relative orientation of the crystals) appear in doublets at slightly lower and higher energy than the isolated molecular level and the energy difference between them is twice the absolute value of the electronic coupling between the two orbitals (a more accurate version of the method was presented in ref. 51). Substantially identical information is provided by band structure computations, ${ }^{52-55}$ which, in the case of organic crystals, can be described analytically as a function of few intermolecular coupling parameters. ${ }^{56}$ Many levels of theory have been used for this computation including extended Hückel, ${ }^{57}$ semiempirical models like INDO $/ \mathrm{S}^{58}$ DFT methods ${ }^{52}$ and tight-binding approximate DFT. ${ }^{59}$

Many systematic investigations on the dependence of the electronic coupling on the molecular structure and relative orientation in the crystal led to the disappointing conclusion that there is no simple rationalization of this parameter. ${ }^{60}$ Frontier orbitals have many nodal planes and small displacement of one molecule with respect to another will lead to a major change in the intermolecular coupling ${ }^{50,61}$ (see also Fig. 2). An experimental manifestation of this fact is the crystallochromy of perylene derivatives ${ }^{62}$ and other crystals ${ }^{63}$ (small changes in the crystal structure affect the intermolecular coupling and the absorption spectrum). Theoretical computations also confirm that the minor changes in the unit cell 


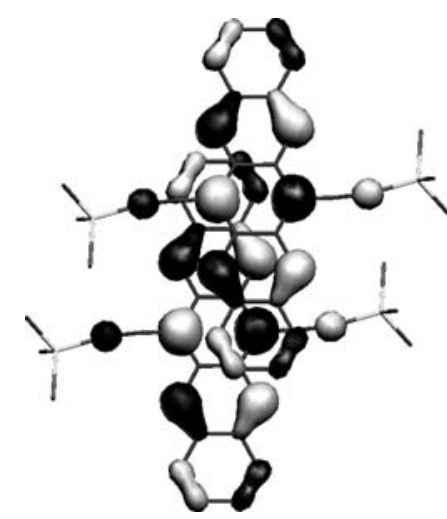

Fig. 2 A representation of the overlap between the HOMO of two TMS pentacene derivatives. ${ }^{61}$ It is clear from the nature of the interacting orbitals (with complex shape and several nodal planes) that a minor displacement of one molecule with respect to the other will change the pattern of the interaction. This appears to be a general characteristic of all relevant intermolecular interactions between large conjugated molecules in a crystal.

recorded for a family of pentacene polymorphs are sufficient to alter considerably the band structure of the material. ${ }^{56}$ This sensitivity of the intermolecular coupling to the geometry means that it is essentially not possible to crystal engineer materials with large coupling and therefore these need to be identified through systematic searches.

Much more 'predictable' is the qualitative behaviour of the intramolecular reorganization energy which is approximately proportional to the inverse of the size of the conjugated portion of the molecule, ${ }^{64,65}$ and increases slightly with the addition of functional polar groups. ${ }^{66}$ This energy is given by $\lambda=E^{+}\left(M^{+}\right)-E^{+}(M)+E(M)-E\left(M^{+}\right)$, where $E(M)$ and $E^{+}\left(M^{+}\right)$are the energies of the neutral and charged species in their optimized geometry, respectively, $E\left(M^{+}\right)$is the energy of the neutral state in the geometry of the charged state and $E^{+}(M)$ the energy of the charged state in the geometry of the neutral. When a more general Hamiltonian including more normal modes per molecule is considered, the contribution of each mode to the reorganization energy needs to be evaluated. The accuracy of these computations has been verified by comparing experimental and theoretically predicted high resolution photoelectron spectra. ${ }^{67}$ In some cases it was possible to observe a correlation between decreasing reorganization energy and increased field effect mobility. ${ }^{68}$ It is generally assumed that the external reorganization energy due to lattice relaxation around the charged molecule is smaller than the intramolecular component, but a revision of the initial estimates ${ }^{69,70}$ may be necessary since the materials with largest mobility have very small reorganization energies and the external contribution may be a significant factor after all (it was recently found, for example, that the nature of the two-dimensional transport at the rubrene-dielectric interface is determined by the polarization properties of the dielectric ${ }^{71}$ ).

All the computational studies described above are extremely valuable for the understanding of the charge transport because, thanks to their extensive validation with spectroscopic measurements and across different research groups, they constitute a robust set of data to be used in conjunction with the experimental results for the development of consistent transport theories.

\subsection{The need of more sophisticated transport theories}

The combined consideration of the experimental evidence, the computational data and the elementary transport theories indicated that the latter are not suitable to describe the charge transport in crystalline semiconductors. The charge mobility of single crystalline samples decreases with increasing temperature $^{14,17}$ apparently in agreement with a delocalized transport mechanism similar to the band conduction in silicon. On the other hand, the mean free path of the charge carrier as deduced by the semiclassical transport theory is too small (few Angstroms) to be consistent with a band transport. An analysis presented by Cheng et al. indicated that band transport models are not tenable above $150 \mathrm{~K},{ }^{58}$ although band-like transport has been reported up to room temperature. The inspection of the data of coupling and reorganization energy for the most conductive solids indicates that localization due the internal reorganization energy is quite unlikely, i.e. the reorganization energy of pentacene or rubrene is too small compared with the intermolecular coupling. In the limited number of cases when the hopping mechanism could be active (e.g., when the reorganization energy is substantially larger as in hexathiophene), the hopping rate equation cannot be described very accurately by the Marcus equation (eqn (5)) and its generalizations, because the condition $|\tau| \ll \lambda$ is not rigorously valid. Many authors have worked on the intermediate regime between band and hopping transport ${ }^{72}$ including the recent proposal by Cheng and Silbey ${ }^{73}$ who used a variational approach to find the best degree of localization of the wavefunction for a perturbative description of the charge dynamics. The transition between band and hopping is predicted by these models to be taking place in a narrow range of temperatures and so they are unable to explain the persistence of band-like mobility and small scattering distances for a very large temperature range.

One of the ingredients missing in the model of eqn (4) is the non-local (or Peierls) electron phonon coupling, i.e. the modulation of the intermolecular coupling by the intra and intermolecular vibrations of the molecule in the crystal. Since, as we have seen in section 2.2, the intermolecular coupling is extremely sensitive to the relative position of the molecules, this coupling is expected to play an important role for the mobility in organic solids. ${ }^{74}$ Hannewald et al. ${ }^{75}$ included this effect in a rather general Hamiltonian whose parameters were computed for naphthalene from first principles. Following the same spirit of the original Holstein paper $^{39}$ they showed that a polaronic band (obtained by renormalizing the purely electronic band) is formed by the carrier dressed with the lattice deformations. Their model correctly reproduces the temperature dependence of the mobility and clearly demonstrates the importance of non-local electron phonon coupling terms. An analogous attempt had been presented by Munn and Silbey $^{76}$ (in the absence of a realistic evaluation of the electron-phonon coupling term) and has been more recently applied by Wang et al. ${ }^{77}$

Band renormalization models always imply the possibility of 'averaging' out the effect of the vibrations. Troisi and Orlandi explicitly computed the time dependent intermolecular coupling in a pentacene crystal and they showed that the fluctuation of the coupling due to thermal motions is of the same order of magnitude of its average value. This computation used classical 


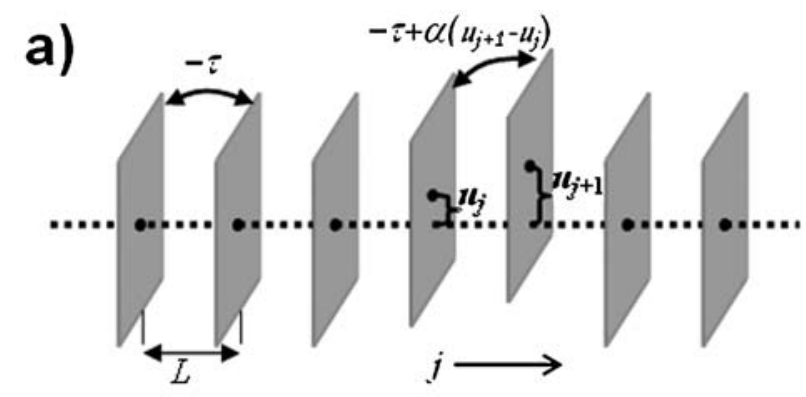

b)

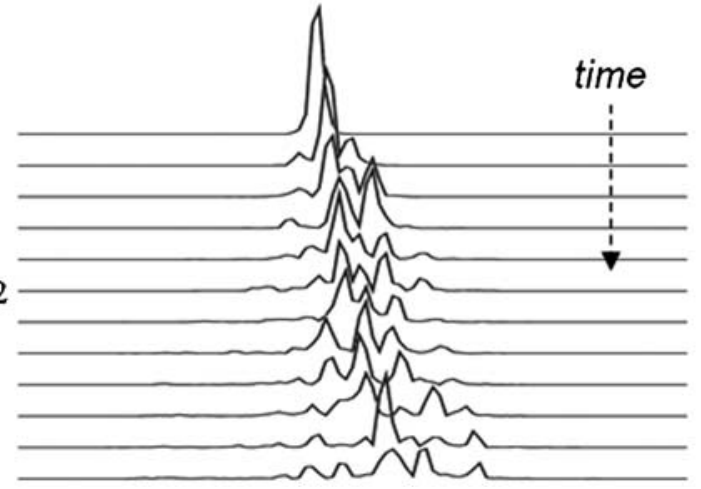

Fig. 3 (a) Scheme of a minimalist model used to describe the charge transport in organic semiconductors: the average coupling between two neighbouring sites is $-\tau$, each site (molecule) $j$ can be displaced by $u_{j}$ from its equilibrium position, and the relative displacement between two molecules $j$ and $j+1$ modulates the intermolecular coupling with the electron-phonon coupling term $\alpha\left(u_{j+1}-u_{j}\right)$. This model can be integrated numerically and (b) shows the typical evolution of the wavefunction from an initial state (localized by disorder). Adapted from ref. 79.

molecular dynamics simulation of the solid, to evaluate its nuclear dynamics, in conjunction with the quantum chemical evaluation of the electronic coupling. ${ }^{78}$ Considering that the correlation among the coupling between different molecules was modest, it was shown that the thermal motions created sufficient disorder in the electronic Hamiltonian to cause localization of the charge carrier. This dynamic localization is quite different from the localization due to the reorganization energy and leads to a completely different carrier dynamics. The latter was explored numerically using a semiclassical model Hamiltonian described in Fig. 3 which allowed the evaluation of the temperature dependent mobility. ${ }^{79}$ By including only the non-local electron phonon coupling due to low frequency vibrations, this transport model, termed diffusion limited by thermal disorder (DLTL), is able to explain the decrease of the mobility with temperature also for temperatures for which the band transport was shown to be inconsistent. In other words, it explains why the transport appears to be 'band-like' also when the electronic bands do not exist because the translational symmetry of the electronic Hamiltonian is destroyed by the thermal motions. In a recent improvement of the same model, which includes also the effect of local electron-phonon coupling, the full set of system parameters was derived computationally for rubrene and the absolute value of the hole mobility was computed without any adjustable parameters (Fig. 4) ${ }^{80}$ The computed mobility values were 3 times larger than the experimental one in the same temperature range, a result that is to be considered rather encouraging.

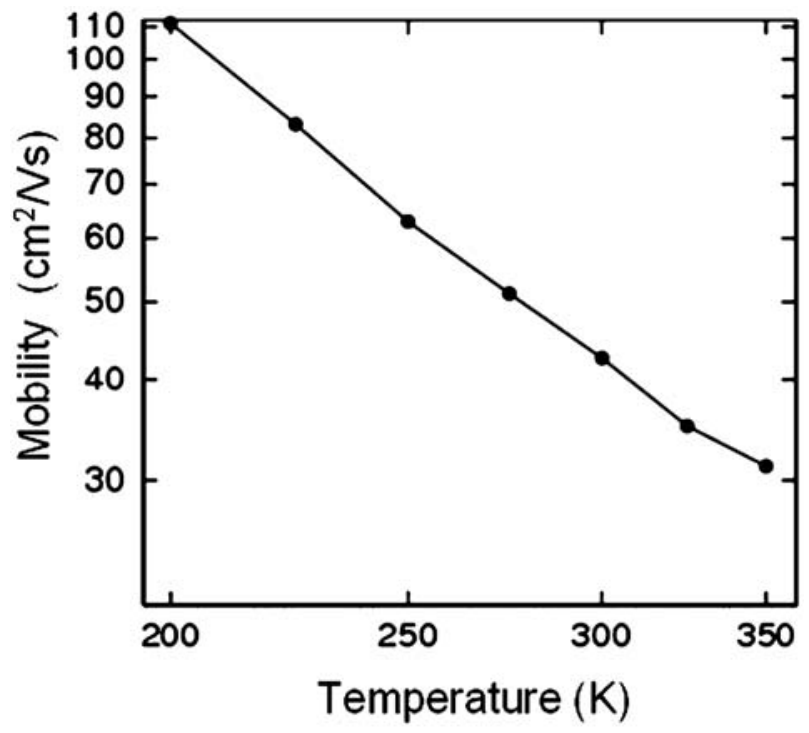

Fig. 4 Absolute value of the hole mobility computed by numerical integration of the semiclassical Hamiltonian with parameters computed for rubrene crystal and no adjustable parameters. ${ }^{80}$ The experimental mobility between 200 and $300 \mathrm{~K}^{25}$ follows the same temperature dependence and is approximately 3 times larger than the computed value.

The ideas that localization theories are relevant for the transport in organic semiconductors was further investigated by Picon et al., ${ }^{81}$ which identified in the fluctuation of the polarization energy an alternative source of disorder in the electronic Hamiltonian.

\subsection{Some new experimental evidences}

As results from different theoretical methods gradually approach those of the experiments, it is important to consider a broader set of experimental measurements that could shed light on the transport mechanism and confirm the prediction of the theories. With particular reference to the new models described in the previous section, it is quite important to retrieve independent information on the nuclear motions of the molecules inside the crystals and their coupling with the electronic degrees of freedom. The existence of relatively large amplitude motions of the molecules inside a bulk crystals was repeatedly shown by crystallographic studies ${ }^{82-84}$ including one on pentacene. ${ }^{85}$ Simple evidence of the strong coupling of these motions with the electronic degrees of freedom was provided by Koch et al. ${ }^{86}$ which studied the electronic structure of a pentacene thin film on graphite revealing a temperature dependent band width. In particular, they observed a reduction of the band width from 240 to $190 \mathrm{meV}$ on increasing the temperature from $120 \mathrm{~K}$ to room temperature, in agreement with the view that at higher temperature the electrons are more localized and therefore easier to remove from the solid. Important evidence that charge carriers in ultrapure pentacene may be localized by disorder was provided by Lang et $\mathrm{al}^{87}$ who analyzed the tail of the density of states using optical (photocurrent) and electrical (FET) methods. They reported a tail of the density of states very similar to those observed in amorphous solid, a fact that supports the 
idea of an intrinsic electronic disorder in the crystal as suggested by the computations.

Another indirect source of information on the density of states in organic semiconductor may be provided by the measurement of the Seebeck coefficient performed recently on a series of organic devices by Pernstich et al. ${ }^{88}$ When two ends of a sample are held at slightly different temperatures the thermoelectric voltage between these can be related to the energy dependent electric conductivity (the Seebeck coefficient is the ratio between this voltage and the temperature difference). The authors find that the Seebeck coefficient in a series of organic samples behaves similarly to that of inorganic semiconductors, i.e. as in a system characterized by band-like transport with exponentially distributed in-gap trap states and no contribution from small polaron formation. Indication of band-like transport was also observed by Li et al. ${ }^{89}$ who measured the integrated optical conductivity in rubrene single crystal (at low temperature) and the measured mobility anisotropy compared favourably with the inverse effective mass tensor computed from band theory.

A potentially very useful tool to investigate the coupling between nuclear and electronic motions is the measurement of $\mathrm{THz}$ conductivity. Band-like mobility was reported in several papers ${ }^{90,91}$ but the greater interest toward this techniques relies in the possibility to explore the effect of the soft vibrational modes (in the $0.8-1.6 \mathrm{THz}$ region), which are predicted to have the largest coupling with the electron dynamics. H. Van Laarhoven et $a .^{92}$ recently measured a transient absorption of radiation at 1.1 THz upon photoexciting a pentacene sample. This signal was interpreted as deriving from the vibrational modes that modulate the intermolecular electronic coupling which are activated by the photoexcitation in analogy with the effect of soliton induced infrared absorption. Localization of the charge carriers was recently investigated also via EPR by Marumoto et al. ${ }^{93}$ who found localization to within $\sim 10$ molecules at room temperature, in agreement with the model of ref. 8.

It needs to be stressed that the localization length of the carrier may depend on the characteristic timescale of the experimental techniques. The general theoretical problem is to understand how the interaction between the nuclear and the electronic degrees of freedom determines the charge localization and dynamics and different timescales.

\section{Modelling charge transport in soft matter}

While there has been much work on the modelling of charge transport properties in molecular semiconductors, the most promising technological applications of organic electronics may utilise soft materials, such as liquid crystals (LC) ${ }^{23}$ or polymers. ${ }^{94}$ Despite typically having lower charge mobilities than solid state semiconductors, their ability to self-organise into complex supramolecular structures makes them easier to process than their solid-state cousins.

Understanding the charge transport properties in such systems requires knowledge on a number of levels. Some of the parameters that determine charge transport may be found from the electronic structure around single molecules, which may be studied accurately using high-level quantum chemical calculations. In condensed phases this electronic structure, and hence charge transport properties, becomes dependent on the molecular environment. This microstructure formed from a few hundreds of molecules (or monomers in the case of polymeric materials) may be investigated using atomistic molecular dynamics (MD) simulations. Finally larger scale structures, consisting of many hundreds of molecules, may be studied with coarse-grained simulations using simplified, generic potentials. To investigate the actual charge transfer in such large scale structures, kinetic (or hopping) MC simulations may be used.

Charge transport in soft materials is strongly influenced by both static and dynamic disorder. In semiconducting and conducting polymers, $\pi$-electron delocalization along the polymer backbone gives rise to charge transport. Deviations from planarity in the backbone (particularly in polymers containing aromatic rings) caused by conformational disorder leads to decreases in the charge mobility along polymer chains. ${ }^{95}$ Likewise fluctuations in the interchain spacing in polymer crystals leads to decreases in the (already relatively low) interchain charge transfer. In the columnar phases of discotic liquid crystals, changes in the separation between molecules in the columns, lateral disorder, and changes in the relative angle between neighbouring molecules in a column may significantly affect the values of the transfer integral, hence charge mobility. ${ }^{96}$

As for crystalline molecular semiconductors the parameters governing charge transport may be determined from quantum mechanical calculations. These depend on molecular conformation and local ordering, so the actual charge mobility at finite temperature depends on the complex interplay between nuclear and electronic degrees of freedom. For molecular organic semiconductors in the crystalline phase the exploration of the nuclear dynamics is relatively straightforward since the harmonic approximation for the nuclear modes can be made and a model Hamiltonian can be easily built. As polymers and LCs possess instead many more degrees of freedom, the number of configurations that need to be sampled is much greater (and the most likely configurations are not necessarily those that optimize charge transfer), and they are difficult to represent using simplified models. The coupling between nuclear and electronic degrees of freedom is therefore particularly difficult to deal with. While modelling on a single level (e.g. electronic structure calculations, molecular simulations) can give valuable insight into the properties of soft materials, by necessity it only gives part of the picture. Thus there is currently much interest in multiscale methods which combine calculations across a range of length and time scales including both the electron and the nuclei dynamics.

\subsection{Semiconducting liquid crystals}

Traditionally LCs have been divided into two classes depending on their molecular shape; either rod-shaped (calamitic LCs) or disc-shaped (discotic LCs, DLC). ${ }^{97}$ Molecules belonging to these different classes form different phases. Calamitic molecules form nematic phases, with liquid like spatial order but long-range orientational order, with the long molecular axes aligned, and smectic phases where the molecules assemble into layers (1-D crystals). Alongside nematic phases, discotic molecules also exhibit columnar phases where the molecules form into columns 
(a)

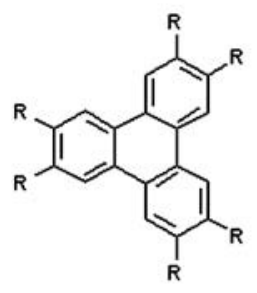

(b)

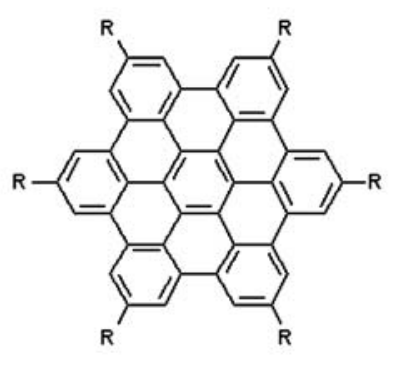

(c)

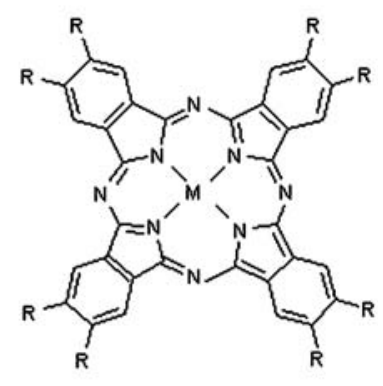

$\mathrm{M}=$ metal or $2 \mathrm{H}$

Fig. 5 Structures of typical core units for semiconducting discotic liquid crystals (a) triphenylene, (b) hexaalkyl hexa-peri-hexabenzocoronene (HBC), and (c) phthalocyanine (Pc).

that assemble into regular (typically hexagonal) arrays. ${ }^{98}$ Semiconducting DLCs typically contain rigid aromatic cores which assemble into columns with favourable $\pi-\pi$ stacking between the cores making them promising candidates for use in organic electronics and photovoltaics. ${ }^{23,98}$ Common examples of these include derivatives of triphenylene, ${ }^{99,100}$ hexabenzocoronene (HBC), ${ }^{101}$ and phthalocyanine ${ }^{102,103}$ (see Fig. 5), many of which have been tested in devices. Experimental studies have studied the effect of aromatic core size ${ }^{104}$ and side-chains ${ }^{105}$ on phase behaviour $^{102,103}$ and charge mobility. ${ }^{106,107}$ Despite the wealth of experimental studies a detailed microscopic understanding of the charge mobility in discotic materials is still lacking. In particular, the complicated interplay between the electronic and nuclear degrees of freedom makes developing structureproperty relationships difficult. One example of this is in the charge mobility in DLCs, where experimentally small changes in the molecular structure, e.g. lengthening of alkyl side chains, may dramatically change the temperature dependence. ${ }^{108}$

Despite their many potential applications of discotic LC, until recently, atomistic simulations had focused on calamitic LCs. ${ }^{109}$ Typical DLCs are significantly larger than typical calamitic LCs, hence their simulation entails a larger computational burden. Thus early atomistic studies considered small systems of discotic molecules in the isotropic phase ${ }^{110}$ or over short timescales. ${ }^{111}$ However, in the interim a number of simulation studies were performed using idealised models, such as the Gay-Berne (GB) potential. ${ }^{112}$ MC simulations of GB discs have shown the formation of a range of phases including nematic and the technologically important columnar mesophases, alongside crystalline solid and isotropic liquid phases. In particular, the stability of the columnar phase is strongly influenced by the strength of the face to face interaction between the discs. ${ }^{113}$ Considerable attention has also been paid to the effect of electrostatic interactions on the stability of the columnar phase. ${ }^{114-116}$

In order to study real materials it is necessary to move beyond these simple idealised models and consider atomically detailed models. Recent increases in computational resources have now made such simulations a realistic proposition. Initial simulations were performed on single columns. ${ }^{117}$ Atomistic Monte Carlo (MC) simulations have also been used to study the effect of substitutions on the structure of column in semiconducting hexaalkoxy triphenylenes. ${ }^{118} \mathrm{MD}$ simulations of systems with many columns have studied the microscopic structure and dynamics in the hexagonal columnar phase. These have shown only weak coupling between molecules in different columns and collective diffusion within the columns. ${ }^{119,120}$ The conformations of side chains attracted particular attention, because, while these can have little affect on the electronic structure of such molecules, they can strongly influence the phase behaviour. A recent study of HBC derivatives $^{120}$ in hexagonal columnar and rectangular crystal phases has shown dramatic differences in the order and diffusion in these systems, particularly with bulk side chains such as those with branched alkanes or phenyl rings. It should be noted that only recently has the transition from isotropic liquid to the columnar phase been simulated using atomistic MD. ${ }^{121}$ The required simulation time was of the order of $100 \mathrm{~ns}$ (compared to $10 \mathrm{~ns}$ for the isotropic-nematic transition in calamitic $\mathrm{LCs}^{122}$ ), which illustrates the long timescales needed for the simulation of DLCs.

As for solid crystalline semiconductors, the parameters relevant for the charge transport properties may be obtained directly from quantum chemical calculations. One of the first such studies on DLCs ${ }^{96}$ presented the evaluation of the charge mobility in triphenylene using parameters determined from band structure calculations. The temperature dependence of the charge mobility was found to differ depending on the strength of the fluctuations - at low fluctuation strength the mobility was found to be almost independent of temperature, in agreement with experimental results. ${ }^{108,123}$ For stronger fluctuations the mobility becomes temperature dependent and of much higher magnitude compared to experiment. Later work $^{124}$ used the same model, but with parameters derived from DFT calculations, to study charge transfer in triphenylene derivatives. Bredas and coworkers have theoretically studied the transfer integrals and reorganization energies for DLCs, ${ }^{125-127}$ in particular the effect of changing molecular positions (lateral disorder within columns) ${ }^{125}$ and changing molecular structure. ${ }^{126}$

Due to the many different length and timescales involved in charge (and exciton) transfer in DLCs, this may be effectively studied using multiscale methods. ${ }^{128}$ Some early work, performed by Bacchiocchi and Zannoni, combined molecular MC simulations of a model DLC in the columnar phase with kinetic MC simulations of the energy transfer. ${ }^{129}$ The LC 


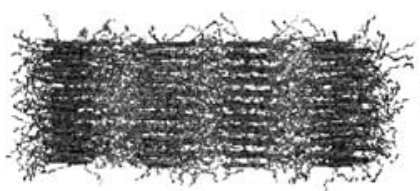

(a)

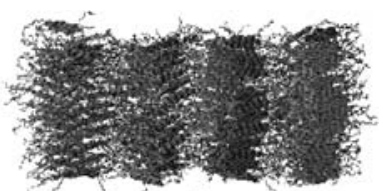

(b)
Fig. 6 MD simulation snapshots of columns of $\mathrm{HBC}$ molecules with (a) $C_{12}$ and (b) $C_{10-6}$ side chains. Used with permission from ref. 131. (Copyright (2008) by the American Physical Society.)

molecules were modelled as GB discs, with the energy transfer between them being modulated by their separation and relative orientations, modelled using a MC approach. Transport along columns was enhanced by increasing the molecular width. The same approach used in this study has recently been applied to study transport in molecular glasses. ${ }^{130}$

More recently, atomistic MD simulations, quantum chemical calculations and kinetic MC simulations have been combined to study charge transfer in $\mathrm{HBC}$ derivatives by Kirkpatrick et al. ${ }^{131}$ MD simulations were used to study the local ordering within the columnar phase. Pairs of molecules taken from these simulations were used to calculate the electronic coupling, using quantum chemical calculations at the semi-empirical ZINDO level. The use of many different configurations taken from a long MD trajectory (100 ns) allowed the comprehensive sampling of molecular conformations. Also the conformations used are more representative of those found at finite temperatures in real materials. The MD configurations were also periodically replicated and used to define a lattice for the kinetic MC simulations. The hopping rates between the molecular sites in these simulations were found using the Marcus theory, using the QC electronic coupling. The charge mobilities calculated from these simulations were in good agreement with those measured experimentally, validating the multiscale methodology used. Mobilities for $\mathrm{HBC}$ derivatives with linear side-chains are significantly higher than those with branched side-chains or side-chains containing phenyl-rings. These bulkier side-groups disrupt the local ordering within columns (see Fig. 6). This disorder is also seen in the distribution of transfer integrals $\tau$ : the narrow distribution of $\tau$ for the linear side-chains is broadened for the phenyl-ring derivative, while, for the branched derivative, defects within the columns gives rise to a second peak in the distribution at low values of $\tau$. This thorough investigation indicates clearly that the structure property relation cannot be derived from computations on few idealized structures. This approach is not limited to liquid crystals and it has been recently adopted for the study of the amorphous and crystalline phase of tris(8-hydroxyquinoline) aluminium (Alq3). ${ }^{132}$

\subsection{Polymeric semiconductors}

Organic semiconducting polymers are often characterized by a $\pi$-conjugated backbone as illustrated by the examples in Fig. 7 . While the performance of these materials is often poor in comparison to silicon and other inorganic semiconductors, they combine their electronic properties with ease of processing and flexibility typical of polymers. Charge transport in semiconducting polymers is sensitive to conformational disorder in the polymer backbone $e^{133,134}$ and to chemical

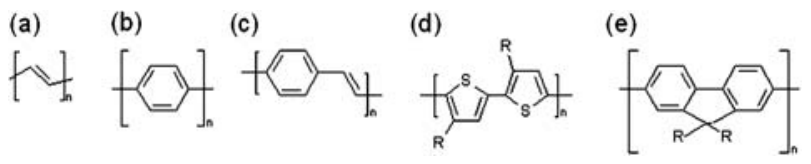

Fig. 7 Structures of typical polymer semiconductors: (a) polytransacetylene (PA), (b) polyparaphenylene (PPP), (c) polyparaphenylenevinylene (PPV), (d) poly(3-alkylthiophene) (P3AT), and (e) polyfluorene (PF).

defects. ${ }^{95,135}$ The charge mobility in polymer semiconductors is also strongly dependent on morphology, both at molecular level and over larger scales. ${ }^{136}$ Both charge transfer between polymer chains ${ }^{36}$ and the persistence length, are dependent on the molecular packing. The need to optimize the chain packing often runs counter to other considerations. In order to ease processing, alkyl side chains are typically added in order to reduce melting temperatures and improve solubility. P3HT and other semiconducting polymers with side chains, form a lamella structure ${ }^{137}$ with two-dimensional conduction within the conjugated sheets, with much reduced charge transfer between them. ${ }^{36}$ The effect of molecular packing may also be seen by comparing regioregular (RR) and regiorandom (rR) P3HT. RR-P3HT has charge mobility several orders of magnitude larger than rR-P3HT, due to its efficient packing and higher interchain overlap. ${ }^{138,139}$ On a larger scale polymer semiconductors often consist of crystalline domains separated by amorphous regions. Molecules in low molecular weight (MW) films are able to form well ordered crystals more easily than those in high MW films. Paradoxically higher charge mobility has been observed in high MW P3HT films, ${ }^{140}$ and this has been rationalized as long polymer chains are able to bridge between different crystalline domains. ${ }^{141}$

A number of experimental techniques have been applied to the study of the structure and charge mobility in polymer semiconductors. The morphology has primarily been studied using atomic force microscopy (AFM), which is only able to probe the surface of the sample, and X-ray diffraction (XRD) ${ }^{142}$ or scattering. ${ }^{143}$ However, X-ray measurements are largely limited to studying the crystalline regions of polymer films, while the amorphous regions are typically large enough to impact charge transport. The charge mobility may be probed by time-of-flight (TOF) measurements, ${ }^{144}$ pulse-radiolysis time-resolved-microwave-conductivity (PR-TRMC), ${ }^{145}$ and modelling space-charge-limited-current (SCLC) diodes ${ }^{146}$ or TFTs. ${ }^{147,148}$ Typically however, TOF and PR-TRMC measurements are dominated by the fastest charge carriers and measurements from SCLC diodes and TFTs may significantly differ from each other. ${ }^{136}$ These limitations in determining both structure and mobility make finding relationships between microscopic structure and charge transport properties difficult and make particularly important the development of computational methodologies able to model in a consistent way heterogeneous experimental data across different materials and morphologies.

Different properties of polymer semiconductors have been studied using quantum mechanical and molecular simulation methods. Due to the interest in the crystalline states of these systems, methods developed for ab initio calculations in solid state physics, such as plane-wave pseudopotential calculations, ${ }^{149}$ may be employed, as well as traditional quantum chemical methods. ${ }^{150}$ These have often been applied to study 
the optical and transport properties of simple examples of $\pi$-conjugated polymers, principally trans-polyacetylene (PA) ${ }^{151,152}$ and poly-para-phenylene (PPV). ${ }^{153,154}$ Recently Street et al. used DFT calculations to derive a transport model for polycrystalline polythiophene combining DFT calculations of the electronic structure of the crystalline domain with a model of interactions at grain boundaries. ${ }^{155}$ Classical MD simulations have also been employed to study the structural properties of conjugated polymers in the crystal phase. In a series of papers the structure of poly(3-butylthiophene) (P3BT) has been investigated as a function of temperature and pressure using atomistic MD simulations and static lattice (SL) calculations. ${ }^{156-159}$ The experimentally observed thermochromism $^{160,161}$ in these materials was explained by a combination of backbone and side-chain torsions (the polymer backbone was also found to planarize under high pressures ${ }^{162}$ ).

More recently, MD simulations and quantum chemical calculations have been combined to study the microstructure and optical properties of semi-conducting polymers in amorphous and crystalline states. In MD simulations of substituted $\mathrm{PPV},{ }^{163}$ the main chains were found to form helices or zig-zags containing quasi-planar segments of 2-4 monomers. Simple quantum mechanical calculations were used to investigate the optical properties using configurations from the MD trajectory. Atomistic simulations have also been used to investigate the optical properties of amorphous polythiophene. ${ }^{164}$ The elongated chains were seen to align, with nearby thiophene rings on different chains lying parallel to each other, but displaced from ideal $\pi$-stacking. Simulations of substituted polythiophenes ${ }^{165}$ have shown that, in the liquid phase, the helical structure seen in the gas phase is suppressed for linear side-chains, but remains for branched ones. In the crystalline phase due to packing constraints, it was found that only a small number of helix defects are possible, and an arrangement of two nearby parallel polymer chains, a possible precursor of an excimer, was found to be unfavourable.

While accurate quantum mechanical calculations are only applicable to small systems or systems with translational symmetry (e.g. crystals), they may be used to provide parameters for semiempirical calculations that may be applied to larger systems. Using a tight-binding model, with some parameters derived from AM1 calculations (the others were fit to experimental data), Yu et al. considered the effect of torsional angle fluctuations on charge mobility in conjugated polymers. ${ }^{166,167}$ In one-dimension the model used was solved analytically, while for the three dimensional case the master equation was solved numerically. The mobility in the one and three dimensional models becomes the same in the high field region, with deviations between the two at low fields. While this model reproduced the experimentally observed field and temperature dependence of the charge mobility, the use of fitted parameters limited its application. The effect of ring torsions on charge mobility in PPV was recently studied using a semi-classical model by Hultell and Stafstrom. ${ }^{168}$ The nuclear motions were treated using a simple force-field, while the electronic degrees of freedom (only the $\pi$-electrons were explicitly considered) were treated within a tight-binding approximation. The model parameters and relaxed geometry of the PPV monomer where determined using DFT calculations. Increases in inter-ring torsions lead to a change in the polaron motion from adiabatic drift to hopping transport. Hopping transport also arises with random torsions at zero applied field, with drift motion restored above a critical field value.

A similar tight-binding approach has been used by Siebbeles and co-workers. ${ }^{169,170}$ Initially, this was used to study charge mobility in PPV and P3HT. The transfer integral between two neighbouring sites (corresponding to monomer units) was modulated by the torsional angle between the sites. These were distributed according to a Boltzmann distribution and held fixed during the simulation. Both the transfer integral and torsional potential were found using quantum chemical calculations at the MP2 level. The time evolution of the meansquared displacement (MSD) of the charge carriers was found to follow two stages. At short times the MSD increases rapidly due to fast delocalization through planar segments, while at longer times the mobility drops due to holes being trapped at sites with large torsional angle differences. P3HT was found to have a mobility $\sim 30$ times smaller than PPV; its weaker torsional potential leading to a higher degree of conformational disorder. Comparison between the experimentally measured charge mobilities and those calculated for finite length chains suggests that the effective length of conducting segments in these polymers contain 100-200 monomers.

This model has been extended to include the effect of dynamic torsional disorder. ${ }^{171,172}$ The time evolution of the torsional angles was modelled using a Fokker-Planck equation, so both deterministic and stochastic time evolution were considered. This model suggests that the main determining factor of the charge mobility is the presence of dynamic disorder - the mobility being increased by a factor of 10-50 compared to static disorder only. This is because the rotation of torsional angles relaxes barriers between monomers, decreasing the time charge carriers are trapped at sites with large torsional angle differences. By contrast, the mobility is only weakly dependent on the torsional rotation rate (decreasing torsional rotation time by a factor of 4 only increases the mobility by $\sim 1.5$ ). Introducing random fluctuations in the site energies and transfer integrals (to model the effect of other vibrational modes) also had a weak effect on the mobility. Applying this model to PPV-derivatives showed that mobility increases with conjugation fraction and chain length. Likewise, studies of alkoxy-substituted PPV showed that the charge mobility was almost linear in the transfer integral, suggesting band-like transport. On going from 0 to 2 alkoxy groups, the charge mobility decreases due to a decrease in transfer integral and torsional rotation rate.

As it can be easily seen from these last examples, the advancement in the modelling methodology for soft matter semiconductors mirrored the same development which took place for molecular crystalline semiconductors. The static picture has been gradually replaced by a dynamic picture where the nuclear motion at different time-scales determines the charge carrier dynamics of the material. A common theme of the methodologies proposed in ref. 169-172 is that it is not possible to 'average' the effect of the nuclear motions without losing an important element of the charge dynamics and, as a consequence, the quality of the overall description stems from 
a balanced computational treatment of electronic and nuclear degrees of freedom, accompanied by a suitable theoretical methodology.

\section{Outlook}

Although we are yet unable to predict quantitatively the charge transport characteristic of organic semiconductors, major progress have been made in the past few years thanks to the combination of new experimental measurements, computational investigations and theories. Several limitations of the theories developed for inorganic semiconductors when applied to organic matters have been discovered and it is now generally acknowledged that new paradigms are necessary to understand organic electronics materials and to improve systematically their quality. The charge mobility in organic solids, as in any other solids, depends on the complicated interaction between nuclear and electronic degrees of freedom, but the effect of nuclear dynamics is much more difficult to treat in molecular and especially in soft materials whose nuclear dynamics is characterized by a multitude of timescales.

It is possible to outline the future challenges for the modelling of organic semiconductors grouping them according to the characteristic time scale involved. Considering the shorter time scale $\left(10^{-14}-10^{-12} \mathrm{~s}\right)$, we are still unable to describe in a consistent way the time evolution of the charge carrier's wavefunction. In particular, we cannot predict the extent of the charge carrier localization because several concurrent effects may takes place: (i) small polaron formation due to intramolecular reorganization, (ii) localization by dynamic and static (in polymer) disorder, (iii) stabilization of the small polaron at the interface with the dielectric and other polarization effects, (iv) trapping by impurities. As we have seen in this perspective, many researchers have stressed the importance of these effects and it is now necessary to compare more systematically their relative importance. Quantum chemical methods are expected to play a major role (especially for the issues (i)-(iii)) to avoid the development of models overloaded with adjustable parameters.

Many different theoretical works indicated that the nuclear dynamics in an intermediate time scale $\left(10^{-12}-10^{-9} \mathrm{~s}\right)$ is strongly coupled to the charge mobility. While nuclear dynamics in molecular crystal can be studied very easily, atomistic simulations of other more complicated organic semiconductors have become possible only in the recent years. The difficulties of these large scale simulations suggest that the collaboration between experts in soft-matter simulations and quantum dynamics can be particularly fruitful in this area.

Finally, many materials proposed for technological application (polymers and liquid crystals) undergo large structural fluctuations in very slow timescales ( $\gg 10^{-9} \mathrm{~s}$ ), sometimes even slower than the typical times of circuit operation. These motions are not directly coupled to the charge carrier but the charge mobility of the material can be indeed modulated by them. Grain boundaries and interfaces involving polymeric crystalline phases are thought to be particularly important for transistor and photovoltaic applications and their effect is essentially unknown at the moment. It would be therefore particularly useful to combine the study of slow dynamics and interfaces in soft-matter with the methodology used in the modelling of mesoscopic electric devices.

\section{Acknowledgements}

We gratefully acknowledge EPSRC for supporting this research.

\section{References}

1 D. Eley, Nature, 1948, 162, 819-819.

2 J. H. Burroughes, D. D. C. Bradley, A. R. Brown, R. N. Marks, K. Mackay, R. H. Friend, P. L. Burns and A. B. Holmes, Nature, 1990, 347, 539-541.

3 J. R. Sheats, H. Antoniadis, M. Hueschen, W. Leonard, J. Miller, R. Moon, D. Roitman and A. Stocking, Science, 1996, 273, 884-888.

4 M. A. Baldo, D. F. O'Brien, Y. You, A. Shoustikov, S. Sibley, M. E. Thompson and S. R. Forrest, Nature, 1998, 395, 151-154.

5 J. H. Burroughes, C. A. Jones and R. H. Friend, Nature, 1988, 335, 137-141.

6 G. Horowitz, D. Fichou, X. Z. Peng, Z. G. Xu and F. Garnier, Solid State Commun., 1989, 72, 381-384.

7 M. Granstrom, K. Petritsch, A. C. Arias, A. Lux, M. R. Andersson and R. H. Friend, Nature, 1998, 395, 257-260.

8 P. Peumans, S. Uchida and S. R. Forrest, Nature, 2003, 425, $158-162$.

9 H. Hoppe and N. S. Sariciftci, J. Mater. Res., 2004, 19, 1924-1945.

10 C. Rothe, Laser Photon. Rev., 2007, 1, 303-306.

11 A. P. Kulkarni, C. J. Tonzola, A. Babel and S. A. Jenekhe, Chem. Mater., 2004, 16, 4556-4573.

12 T. W. Kelley, P. F. Baude, C. Gerlach, D. E. Ender, D. Muyres, M. A. Haase, D. E. Vogel and S. D. Theiss, Chem. Mater., 2004, 16, 4413-4422.

13 K. E. Hagen, Organic Electronics, Weinheimm, 2006.

14 W. Warta and N. Karl, Phys. Rev. B, 1985, 32, 1172-1182

15 Y.-Y. Lin, D. J. Gundlach, S. F. Nelson and T. N. Jackson, IEEE Trans. Electron Devices, 1997, 44, 1325-1331.

16 H. Klauk, D. J. Gundlach, J. A. Nichols and T. N. Jackson, IEEE Trans. Electron Devices, 1999, 46, 1258-1263.

17 O. D. Jurchescu, J. Baas and T. T. M. Palstra, Appl. Phys. Lett., 2004, 84, 3061-3063.

18 O. Ostroverkhova, D. G. Cooke, F. A. Hegmann, J. E. Anthony, V. Podzorov, M. E. Gershenson, O. D. Jurchescu and T. T. M. Palstra, Appl. Phys. Lett., 2006, 88, 162101.

19 V. Podzorov, S. E. Sysoev, E. Loginova, V. M. Pudalov and M. E. Gershenson, Appl. Phys. Lett., 2003, 83, 3504-3506.

20 J. E. Anthony, Chem. Rev., 2006, 106, 5028-5048.

21 W. Clemens, I. Fix, J. Ficker, A. Knobloch and A. Ullmann, J. Mater. Res., 2004, 19, 1963-1973.

22 R. J. Kline and M. D. McGehee, Polym. Rev., 2006, 46, 27-45.

23 M. O'Neill and S. M. Kelly, Adv. Mater., 2003, 15, 1135-1146.

24 J. Zaumseil and H. Sirringhaus, Chem. Rev., 2007, 107, 1296-1323.

25 V. Podzorov, E. Menard, A. Borissov, V. Kiryukhin, J. A. Rogers and M. E. Gershenson, Phys. Rev. Lett., 2004, 93, 086602.

26 H. Bassler, Phys. Status Solidi B, 1993, 175, 15-56.

27 S. V. Novikov, D. H. Dunlap, V. M. Kenkre, P. E. Parris and A. V. Vannikov, Phys. Rev. Lett., 1998, 81, 4472-4475.

28 M. Vissenberg and M. Matters, Phys. Rev. B, 1998, 57, 12964-12967.

29 W. F. Pasveer, J. Cottaar, C. Tanase, R. Coehoorn, P. A. Bobbert, P. W. M. Blom, D. M. de Leeuw and M. A. J. Michels, Phys. Rev. Lett., 2005, 94, 206601.

30 A. Salleo, T. W. Chen, A. R. Volkel, Y. Wu, P. Liu, B. S. Ong and R. A. Street, Phys. Rev. B, 2004, 70, 115311.

31 N. F. Mott, Philos. Mag., 1962, 19, 835.

32 B. N. Limketkai, P. Jadhav and M. A. Baldo, Phys. Rev. B, 2007, 75, 113203 
33 L. Li, G. Meller and H. Kosina, Microelectron. J., 2007, 38, $47-51$.

34 T. Kreouzis, D. Poplavskyy, S. M. Tuladhar, M. Campoy-Quiles, J. Nelson, A. J. Campbell and D. D. C. Bradley, Phys. Rev. B, 2006, 73, 235201.

35 S. Athanasopoulos, J. Kirkpatrick, D. Martinez, J. M. Frost, C. M. Foden, A. B. Walker and J. Nelson, Nano Lett., 2007, 7, $1785-1788$.

36 H. Sirringhaus, P. J. Brown, R. H. Friend, M. M. Nielsen, K. Bechgaard, B. M. W. Langeveld-Voss, A. J. H. Spiering, R. A. J. Janssen, E. W. Meijer, P. Herwig and D. M. de Leeuw, Nature, 1999, 401, 685-688.

37 G. M. Wang, J. Swensen, D. Moses and A. J. Heeger, J. Appl. Phys., 2003, 93, 6137-6141.

38 R. J. Kline, M. D. McGehee and M. F. Toney, Nat. Mater., 2006, 5, 222-228.

39 T. Holstein, Ann. Phys., 1959, 8, 325.

40 M. Pope and C. E. Swenberg, Electronic Processes in Organic Crystals and Polymers, Oxford University Press, New York, 2nd edn, 1999.

41 N. W. Ashcroft and D. Mermin, Solid State Phys., Holt Reinhart and Winston, New York, 1976.

42 C. Jacoboni and L. Reggiani, Rev. Mod. Phys., 1983, 55, 645-705.

43 R. Marcus, J. Chem. Phys., 1956, 24, 979.

44 J. Jortner, J. Chem. Phys., 1976, 64, 4860-4867.

45 A. Nitzan, Chemical Dynamics in Condensed Phases, Oxford University Press, New York, 2006.

46 J. L. Bredas, D. Beljonne, V. Coropceanu and J. Cornil, Chem. Rev., 2004, 104, 4971-5003.

47 V. Coropceanu, J. Cornil, D. A. da Silva, Y. Olivier, R. Silbey and J. L. Bredas, Chem. Rev., 2007, 107, 926-952.

48 V. M. Kenkre, J. D. Andersen, D. H. Dunlap and C. B. Duke, Phys. Rev. Lett., 1989, 62, 1165.

49 A. Troisi and G. Orlandi, Chem. Phys. Lett., 2001, 344, $509-518$

50 J. L. Bredas, J. P. Calbert, D. A. Da Silva Filho and J. Cornil, Proc. Natl. Acad. Sci. U. S. A., 2002, 99, 5804-5809.

51 E. F. Valeev, V. Coropceanu, D. A. da Silva, S. Salman and J. L. Bredas, J. Am. Chem. Soc., 2006, 128, 9882-9886.

52 G. A. de Wijs, C. C. Mattheus, R. A. de Groot and T. T. M. Palstra, Synth. Met., 2003, 139, 109-114.

53 S. E. Koh, B. Delley, J. E. Medvedeva, A. Facchetti, A. J. Freeman, T. J. Marks and M. A. Ratner, J. Phys. Chem. B, 2006, 110, 24361-24370.

54 Y. T. Yang, H. Geng, S. W. Yin, Z. G. Shuai and J. B. Peng, J. Phys. Chem. B, 2006, 110, 3180-3184.

55 F. Ortmann, K. Hannewald and F. Bechstedt, Phys. Rev. B, 2007, 75, 195219.

56 A. Troisi and G. Orlandi, J. Phys. Chem. B, 2005, 109, 1849-1856.

57 R. C. Haddon, X. Chi, M. E. Itkis, J. E. Anthony, D. L. Eaton, T. Siegrist, C. C. Mattheus and T. T. M. Palstra, J. Phys. Chem. B, 2002, 106, 8288-8292.

58 Y. C. Cheng, R. J. Silbey, D. A. da Silva Filho, J. P. Calbert, J. Cornil and J. L. Bredas, J. Chem. Phys., 2003, 118, 3764-3774.

59 R. G. Endres, C. Y. Fong, L. H. Yang, G. Witte and C. Woll, Comput. Mater. Sci., 2004, 29, 362-370.

60 G. R. Hutchison, M. A. Ratner and T. J. Marks, J. Phys. Chem. $B, 2005, \mathbf{1 0 9}, 3126-3138$

61 A. Troisi, G. Orlandi and J. E. Anthony, Chem. Mater., 2005, 17, 5024-2031.

62 P. M. Kazmaier and R. Hoffmann, J. Am. Chem. Soc., 1994, 116, 9684-9691.

63 P. Erk, Curr. Opin. Solid State Mater. Sci., 2001, 5, 155-160.

64 A. Devos and M. Lannoo, Phys. Rev. B, 1998, 58, 8236-8239.

65 V. Coropceanu, M. Malagoli, D. A. da Silva Filho, N. E. Gruhn, T. G. Bill and J. L. Bredas, Phys. Rev. Lett., 2002, 89, 275503.

66 H. Y. Chen and I. Chao, Chem. Phys. Lett., 2005, 401, 539-545.

67 M. Malagoli, V. Coropceanu, D. A. da Silva and J. L. Bredas, J. Chem. Phys., 2004, 120, 7490-7496.

68 M. Mas-Torrent, P. Hadley, S. T. Bromley, X. Ribas, J. Tarres, M. Mas, E. Molins, J. Veciana and C. Rovira, J. Am. Chem. Soc., 2004, 126, 8546-8553.

69 I. Vilfan, Phys. Status Solidi B, 1973, 59, 351-360.

70 I. V. Brovchenko, Chem. Phys. Lett., 1997, 278, 355-359.
71 I. N. Hulea, S. Fratini, H. Xie, C. L. Mulder, N. N. Iossad, G. Rastelli, S. Ciuchi and A. F. Morpurgo, Nat. Mater., 2006, 5, 982-986.

72 S. Fratini and S. Ciuchi, Phys. Rev. Lett., 2003, 91, 256403.

73 Y. C. Cheng and R. J. Silbey, J. Chem. Phys., 2008, 128, 114713.

74 R. G. Della Valle, A. Brillante, L. Farina, E. Venuti, M. Masino and A. Girlando, Mol. Cryst. Liq. Cryst., 2004, 416, 145-154.

75 K. Hannewald, V. M. Stojanovic, J. M. T. Schellekens, P. A. Bobbert, G. Kresse and J. Hafner, Phys. Rev. B, 2004, 69, 075211.

76 R. W. Munn and R. Silbey, J. Chem. Phys., 1985, 83, 1843-1853.

77 L. J. Wang, Q. Peng, Q. K. Li and Z. Shuai, J. Chem. Phys., 2007, 127, 044506.

78 A. Troisi and G. Orlandi, J. Phys. Chem. A, 2006, 110, 4065-4070.

79 A. Troisi and G. Orlandi, Phys. Rev. Lett., 2006, 96, 086601.

80 A. Troisi, Adv. Mater., 2007, 19, 2000.

81 J. D. Picon, M. N. Bussac and L. Zuppiroli, Phys. Rev. B, 2007, 75, 235106.

82 J. Harada and K. Ogawa, J. Am. Chem. Soc., 2001, 123, 10884-10888.

83 J. Harada and K. Ogawa, J. Am. Chem. Soc., 2004, 126, 3539-3544.

84 J. N. Moorthy, P. Venkatakrishnan, G. Savitha and R. G. Weiss, Photochem. Photobiol. Sci., 2006, 5, 903-913.

85 S. Haas, B. Batlogg, C. Besnard, M. Schiltz, C. Kloc and T. Siegrist, Phys. Rev. B, 2007, 76, 205203.

86 N. Koch, A. Vollmer, I. Salzmann, B. Nickel, H. Weiss and J. P. Rabe, Phys. Rev. Lett., 2006, 96, 156803.

87 D. V. Lang, X. Chi, T. Siegrist, A. M. Sergent and A. P. Ramirez, Phys. Rev. Lett., 2004, 93, 086802.

88 K. P. Pernstich, B. Rossner and B. Batlogg, Nat. Mater., 2008, 7, 321-325.

89 Z. Q. Li, V. Podzorov, N. Sai, M. C. Martin, M. E. Gershenson, M. Di Ventra and D. N. Basov, Phys. Rev. Lett., 2007, 99.

90 O. Ostroverkhova, D. G. Cooke, S. Shcherbyna, R. F. Egerton, F. A. Hegmann, R. R. Tykwinski and J. E. Anthony, Phys. Rev. $B, 2005,71,035204$.

91 O. Ostroverkhova, D. G. Cooke, F. A. Hegmann, R. R. Tykwinski, S. R. Parkin and J. E. Anthony, Appl. Phys. Lett., 2006, 89, 192113.

92 H. A. van Laarhoven, C. F. J. Flipse, M. Koeberg, M. Bonn, E. Hendry, G. Orlandi, O. D. Jurchescu, T. T. M. Palstra and A. Troisi, J. Chem. Phys., 2008, 129, 044704.

93 K. Marumoto, S. Kuroda, T. Takenobu and Y. Iwasa, Phys. Rev. Lett., 2006, 97, 256603.

94 J. L. Bredas, D. Beljonne, V. Coropceanu and J. Cornil, Chem. Rev., 2004, 104, 4971-5004.

95 A. N. Caruso, D. Q. Feng, Y. B. Losovyj, D. L. Schulz, S. Balaz, L. G. Rosa, A. Sokolov, B. Doudin and P. A. Dowben, Phys. Status Solidi B, 2006, 243, 1321-1330.

96 M. A. Palenberg, R. J. Silbey, M. Malagoli and J. L. Bredas, J. Chem. Phys., 2000, 112, 1541-1546.

97 P. G. de Gennes and J. Prost, The Physics of Liquid Crystals, Claredon Press, Oxford, 2nd edn, 1995.

98 S. Sergeyev, W. Pisula and Y. H. Geerts, Chem. Soc. Rev., 2007, 36, 1902-1929.

99 A. N. Cammidge and R. J. Bushby, in Handbook of Liquid Crystals, ed. D. Demus, J. W. Goodby, G. W. Gray, H. W. Spiess and V. Vill, Wiley-WCH, Weinheim, Editon edn, 1998, vol. 2B, pp. 693-747.

100 A. N. Cammidge, Philos. Trans. R. Soc. London, Ser. A, 2006, 364, 2697-2708.

101 P. Herwig, C. W. Kayser, K. Müllen and H. W. Spiess, Adv. Mater., 1996, 8, 510-513.

102 J. F. Van Der Pol, E. Neeleman, J. W. Zwikker, R. J. M. Nolte, W. Drenth, J. Aerts, R. Visser and S. J. Picken, Liq. Cryst., 1989, 6, 577-592.

103 S. Sergeyev, E. Pouzet, O. Debever, J. Levin, J. Gierschner, J. Cornil, R. G. Aspe and Y. H. Geerts, J. Mater. Chem., 2007, 17, $1777-1784$.

104 M. G. Debije, J. Piris, M. P. deHaas, J. M. Warman, Z. Tomovic, C. D. Simpson, M. D. Watson and K. Mullen, J. Am. Chem. Soc., 2004, 126, 4641-4645.

105 H. Bock, M. Rajaoarivelo, S. Clavaguera and E. Grelet, Eur. J. Org. Chem., 2006, 2006, 2889-2893. 
106 A. M. van de Craats, J. M. Warman, A. Fechtenkötter, J. D. Brand, M. A. Harbison and K. Müllen, Adv. Mater., 1999, 11, $1469-1472$.

107 E. O. Arikainen, N. Boden, R. J. Richard J. Bushby, J. Clements, B. Movaghar and A. Wood, J. Mater. Chem., 1995, 5, 2161-2165.

108 N. Boden, R. J. Bushby, J. Clements, B. Movaghar, K. J. Donovan and T. Kreouzis, Phys. Rev. B, 1995, 52, 13274.

109 M. R. Wilson, Int. Rev. Phys. Chem., 2005, 24, 421-455.

110 A. Maliniak, J. Chem. Phys., 1992, 96, 2306-2317.

111 I. Ono and S. Kondo, Bull. Chem. Soc. Jpn., 1992, 65, 1057-1061.

112 J. G. Gay and B. J. Berne, J. Chem. Phys., 1981, 74, 3316-3319.

113 D. Caprion, L. Bellier-Castella and J. P. Ryckaert, Phys. Rev. E, 2003, 67, 041703.

114 R. Berardi, S. Orlandi and C. Zannoni, J. CHem. Soc., Faraday Trans., 1997, 93, 1493-1496.

115 R. Berardi, S. Orlandi and C. Zannoni, Phys. Chem. Chem. Phys., 2000, 2, 2933-2942.

116 R. Berardi, S. Orlandi and C. Zannoni, Liq. Cryst., 2005, 32, $1427-1436$.

117 F. M. Mulder, J. Stride, S. J. Picken, P. H. J. Kouwer, M. P. de Haas, L. D. A. Siebbeles and G. J. Kearley, J. Am. Chem. Soc., 2003, 125, 3860-3866.

118 L. Muccioli, R. Berardi, S. Orlandi, M. Ricci and C. Zannoni, Theor. Chem. Acc., 2007, 117, 1085-1092.

119 G. Cinacchi, R. Colle and A. Tani, J. Phys. Chem. B, 2004, 108, 7969-7977.

120 D. Andrienko, V. Marcon and K. Kremer, J. Chem. Phys., 2006, 125, 124902.

121 P. L. Cristinziano and F. Lelj, J. Chem. Phys., 2007, 127, 134506.

122 J. A. K. Howard, C. Mcbride and M. R. Wilson, Mol. Phys., 1998, 93, 955-964.

123 A. M. van de Craats, L. D. A. Siebbeles, I. Bleyl, D. Haarer, Y. A. Berlin, A. A. Zharikov and J. M. Warman, J. Phys. Chem. B, 1998, 102, 9625-9634.

124 K. Senthilkumar, F. C. Grozema, F. M. Bickelhaupt and L. D. A. Siebbeles, J. Chem. Phys., 2003, 119, 9809-9817.

125 J. J. Cornil, V. Lemaur, J. P. Calbert and J. L. Brédas, $A d v$. Mater., 2002, 14, 726-729.

126 V. Lemaur, D. A. daSilvaFilho, V. Coropceanu, M. Lehmann, Y. Geerts, J. Piris, M. G. Debije, A. M. vandeCraats, K. Senthilkumar, L. D. A. Siebbeles, J. M. Warman, J. L. Bredas and J. Cornil, J. Am. Chem. Soc., 2004, 126, 3271-3279.

127 X. Crispin, J. Cornil, R. Friedlein, K. K. Okudaira, V. Lemaur, A. Crispin, G. Kestemont, M. Lehmann, M. Fahlman, R. Lazzaroni, Y. Geerts, G. Wendin, N. Ueno, J. L. Bredas and W. R. Salaneck, J. Am. Chem. Soc., 2004, 126, 11889-11899.

128 F. Müller-Plathe, ChemPhysChem, 2002, 3, 754-769.

129 C. Bacchiocchi and C. Zannoni, Phys. Rev. E, 1998, 58, 3237.

130 C. Bacchiocchi, E. Hennebicq, S. Orlandi, L. Muccioli, D. Beljonne and C. Zannoni, J. Phys. Chem. B, 2008, 112, 1752-1760.

131 J. Kirkpatrick, V. Marcon, J. Nelson, K. Kremer and D. Andrienko, Phys. Rev. Lett., 2007, 98, 227402.

132 J. J. Kwiatkowski, J. Nelson, H. Li, J. L. Bredas, W. Wenzel and C. Lennartz, Phys. Chem. Chem. Phys., 2008, 10, 1852-1858.

133 W. R. Salaneck, O. Inganas, B. Themans, J. O. Nilsson, B. Sjogren, J. E. Osterholm, J. L. Bredas and S. Svensson, J. Chem. Phys., 1988, 89, 4613-4619.

134 J. Choi, M. Chipara, B. Xu, C. S. Yang, B. Doudin and P. A. Dowben, Chem. Phys. Lett., 2001, 343, 193-200.

135 D. K. Chambers, S. Karanam, D. Qi, S. Selmic, Y. B. Losovyj, L. G. Rosa and P. A. Dowben, Appl. Phys. A, 2005, 80, 483-488.

136 R. J. Kline and M. D. McGehee, Polym. Rev., 2006, 46, 27-45.

137 R. D. McCullough, Adv. Mater., 1998, 10, 93-116.

138 Z. Bao, A. Dodabalapur and A. J. Lovinger, Appl. Phys. Lett., 1996, 69, 4108-4110.
139 R. Österbacka, C. P. An, X. M. Jiang and Z. V. Vardeny, Science, 2000, 287, 839-842.

140 R. J. Kline, M. D. McGehee, E. N. Kadnikova, J. Liu and J. M. J. Fréchet, Adv. Mater., 2003, 15, 1519-1522.

141 R. J. Kline, M. D. McGehee, E. N. Kadnikova, J. Liu, J. M. J. Frechet and M. F. Toney, Macromolecules, 2005, 38, 3312-3319.

142 M. J. Winokur and W. Chunwachirasiri, J. Polym. Sci., Part B, 2003, 41, 2630-2648.

143 K. E. Aasmundtveit, E. J. Samuelsen, M. Guldstein, C. Steinsland, O. Flornes, C. Fagermo, T. M. Seeberg, L. A. A. Pettersson, O. Inganas, R. Feidenhans'l and S. Ferrer, Macromolecules, 2000, 33, 3120-3127.

144 S. A. Choulis, J. Nelson, Y. Kim, D. Poplavskyy, T. Kreouzis, J. R. Durrant and D. D. C. Bradley, Appl. Phys. Lett., 2003, 83, 3812-3814.

145 R. J. O. M. Hoofman, M. P. de Haas, L. D. A. Siebbeles and J. M. Warman, Nature, 1998, 392, 54-56.

146 L. Bozano, S. A. Carter, J. C. Scott, G. G. Malliaras and P. J. Brock, Appl. Phys. Lett., 1999, 74, 1132-1134.

147 C. Tanase, E. J. Meijer, P. W. M. Blom and D. M. de Leeuw, Phys. Rev. Lett., 2003, 91, 216601.

148 A. Salleo, Mater. Today, 2007, 10, 38-45.

149 M. C. Payne, M. P. Teter, D. C. Allan, T. A. Arias and J. D. Joannopoulos, Rev. Mod. Phys., 1992, 64, 1045.

150 M. B. Sanjio S. Zade, Chem.-Eur. J., 2007, 13, 3688-3700.

151 P. Vogl and D. K. Campbell, Phys. Rev. Lett., 1989, 62, 2012.

152 P. Vogl and D. K. Campbell, Phys. Rev. B, 1990, 41, 12797.

153 P. Gomes da Costa, R. G. Dandrea and E. M. Conwell, Phys. Rev. B, 1993, 47, 1800.

154 G. Zheng, S. J. Clark, S. Brand and R. A. Abram, J. Phys.: Condens. Matter, 2004, 16, 8609-8620.

155 R. A. Street, J. E. Northrup and A. Salleo, Phys. Rev. B, 2005, 71, 165202-165213.

156 J. Corish, D. E. Feeley, D. A. Morton-Blake, F. Beniere and M. Marchetti, J. Phys. Chem. B, 1997, 101, 10075-10085.

157 H. Xie, J. Corish and D. A. Morton-Blake, Synth. Met., 2000, 113, 65-72.

158 H. Xie, S. O'Dwyer, J. Corish and D. A. Morton-Blake, Synth. Met., 2001, 122, 287-296.

159 S. O'Dwyer, H. Xie, J. Corish and D. A. Morton-Blake, J. Phys.: Condens. Matter, 2001, 13, 2395-2410.

160 K. Tashiro, K. Ono, Y. Minagawa, M. Kobayashi, T. Kawai and K. Yoshino, J. Polym. Sci., B, 1991, 29, 1223-1233.

161 H. Tachibana, N. Hosaka and Y. Tokura, Macromolecules, 2001, 34, 1823-1827.

162 J. Mardalen, Y. Cerenius and P. Haggkvist, J. Phys.: Condens. Matter, 1995, 3501.

163 H.-C. Yang, C.-Y. Hua, M.-Y. Kuo, Q. Huang and C.-L. Chen, ChemPhysChem, 2004, 5, 373-381.

164 D. Curcó and C. Alemán, J. Comput. Chem., 2007, 28, 1743-1749.

165 V. Marcon, N. vanderVegt, G. Wegner and G. Raos, J. Phys. Chem. B, 2006, 110, 5253-5261.

166 Z. G. Yu, D. L. Smith, A. Saxena, R. L. Martin and A. R. Bishop, Phys. Rev. Lett., 2000, 84, 721.

167 Z. G. Yu, D. L. Smith, A. Saxena, R. L. Martin and A. R. Bishop, Phys. Rev. B, 2001, 63, 085202.

168 M. Hultell and S. Stafstrom, Phys. Rev. B, 2007, 75, 104304-104307.

169 F. C. Grozema, P. T. van Duijnen, Y. A. Berlin, M. A. Ratner and L. D. A. Siebbeles, J. Phys. Chem. B, 2002, 106, 7791-7795.

170 F. C. Grozema and L. D. A. Siebbeles, Int. Rev. Phys. Chem., 2008, 27, 87-138.

171 P. Prins, F. C. Grozema and L. D. A. Siebbeles, J. Phys. Chem. B, 2006, 110, 14659-14666.

172 P. Prins, F. C. Grozema and L. D. A. Siebbeles, Mol. Simul., 2006, 32, 695-705. 